\title{
Staged inset of free flaps for complex microsurgical head and neck reconstruction to ensure total flap survival
}

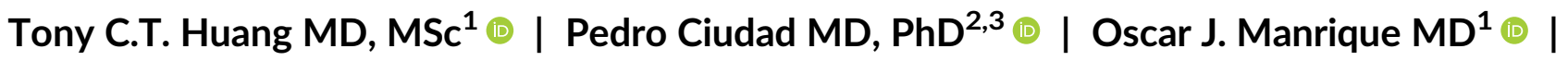 \\ Mouchammed Agko MD ${ }^{2} \odot$ | Shih-Heng Chen MD $^{4}$ | Phoebe Yueh-Bih Tang MD, PhD ${ }^{5}$ | \\ M. Diya Sabbagh MD $^{1}$ () | Hung-Chi Chen MD, PhD, FACS ${ }^{2}$
}

${ }^{1}$ Division of Plastic Surgery, Mayo Clinic, Rochester, Minnesota

${ }^{2}$ Department of Plastic and Reconstructive Surgery, China Medical University Hospital, Taichung, Taiwan

${ }^{3}$ Department of Biological Science and Technology, China Medical University, Taichung, Taiwan

${ }^{4}$ Department of Plastic and Reconstructive Surgery, Chang Gung Memorial Hospital, Chang Gung University, College of Medicine, Taoyuan City, Taiwan

${ }^{5}$ Department of Plastic Surgery, Far Eastern Memorial Hospital, New Taipei City, Taiwan

Correspondence

Hung-Chi Chen, Department of Plastic and

Reconstructive Surgery, China Medical University Hospital, Taichung, Taiwan. Email: d19722@mail.cmuh.org.tw

\begin{abstract}
Introduction: Microsurgical reconstruction of complex head and neck defects often requires reliable tissue in multiple spatial configurations to achieve good functional and aesthetic outcomes. In addition to robust perforators, flap inset and wound closure require great caution to minimize complications especially in the presence of trismus with limited space for inset. This report describes the technique and results of our staged approach to flap inset which increases the chance of total flap survival in complex head and neck reconstructions.

Methods: From February 2010 to August 2016, a total of 53 patients were identified via a retrospective chart review. Patients who were suspected to have a high risk of vascular compromise and subsequently underwent complex head and neck reconstruction with staged inset of anterolateral thigh (ALT) flaps were included. The flap was inset only partially at the recipient site initially with fewer sutures. At a second stage, after neovascularization from surrounding tissue was established, the flap was partially elevated, divided and mobilized on its own pedicle for definitive inset.

Results: Fifty-one patients had complete survival of flaps and had uneventful postoperative course. Six cases required immediate re-exploration to release wound tension or reposition the pedicle. Of these, 4 flaps were salvaged, 2 failed due to small perforators despite anastomosis revision. The flap survival rate was $96.2 \%$ and the average time between 2 stages was 24 days (range, 21-28).

Conclusion: Staged flap inset can improve free flap survival in complex head and neck reconstructions.
\end{abstract}

\section{1 | INTRODUCTION}

A successful complex, 3-dimensional head and neck microsurgical reconstruction depends on the reliable inset of the components of the flap (skin, muscle, oral lining, and bone) and their proper spatial orientation and volume (Chana \& Wei, 2004; Huang, Chen, Wei, Cheng, \& Schnur, 2003). Wide local resection of advanced stage tumors may result in extensive composite defects involving the skin, soft tissue, oral lining, and bone. Such defects require large composite flaps for reconstruction. Multiflap approaches (Huang et al., 2003; Jeng, Kuo, Wei, Su, \& Chien, 2005; Kuzon, Jejurikar, Wilkins, \& Swartz, 1998) have been reported for this purpose, however, additional donor sites and vascular anastomoses, lead to increased donor site morbidity and operative times (Huang et al., 2002, 2003; Gong et al., 2015). Furthermore, the reconstruction may be rendered even more challenging by previous failed attempts at reconstruction. Radiotherapy is another confounding factor that impairs vascular function (Halle et al., 2009) it induces the formation of fibrous scar tissue at the recipient site (Salgado et al., 2002) and produces trismus of the mouth that results in limited space for free flap inset (Louise Kent et al., 2008; Wang et al., 2005). Altogether, these factors may consequently increase the chance of free flap failure (Halle et al., 2009; Khouri et al., 1998; Pohlenz et al., 2007). 
Tension-free wound closure after free flap transfer is essential for flap survival. During inset, circulatory disturbances may arise within the flap due to excessive folding of the flap in an attempt to fit it into a limited 3-dimensional space. This type of inset can cause torsion and tension on the whole flap or its components, resulting in partial or total failure. In addition, the normal swelling can cause an obstruction of the venous outflow with a subsequent arterial inflow disturbance.

A single-stage flap division and inset is usually the preferred method in most reconstructions as it is more time and cost-efficient. Careful flap planning in a single-stage free flap reconstruction can minimize vascular complications. Yet, certain high-risk patients, particularly those with recurrent cancer and had undergone multiple tumor resections and adjuvant radiotherapy are still at risk of flap compromise. Therefore, such patients are poor candidates for a single-stage approach. A more conservative approach, with a higher safety margin is needed in these patients. The goal of this report is to introduce the staged flap inset, as an alternative to the conventional single-stage approach, for complex head and neck reconstruction with the goal of improving the chance of total flap survival. We also report the results from our experience.

\section{PATIENTS AND METHODS}

This was an institutional review board approved, single-center, retrospective chart review of patients who underwent staged head and neck reconstruction using the anterolateral thigh (ALT) flap. From February 2010 to August 2016, 53 patients were identified. There were 42 males and 11 females with an average age of 65 years (range, 45-72) (Table 1). All surgeries were carried out by the senior author (HCC). There were 40 cases with ALT, 13 cases with ALT plus a muscle component (either vastus lateralis or TFL). Oral cancer resection was the reason for reconstruction in all patients in this cohort. Most patients had history of betel quid chewing ( $n=43$ ), alcohol consumption ( $n=37$ ), and cigarette smoking $(n=49)$. Forty-one patients had previous surgical procedures and 35 received preoperative radiotherapy (Table 1). The recipient arteries used were: transverse cervical $(n=19)$, superior thyroid $(n=30)$, and thoracoacromial $(n=4)$ arteries. The recipient veins used were: external jugular vein $(n=42)$ and branches of the internal jugular vein $(n=11)$ (Table 1$)$.

The decision to implement a staged inset was based on the following factors: the number and size of perforators, the spatial orientation of the defect, risk of infection (intraoral defects), preoperative radiotherapy, recipient site scarring, previous reconstructive attempts, and presence of peripheral vascular disease. Moreover, a primary inset was often attempted initially and switched to a staged inset due to signs of vascular compromise such as flap congestion and ischemia.

\section{1 | Surgical technique}

During the first stage of the procedure, all perforators were properly marked on the skin with non-absorbable sutures, allowing easy identification of each perforator in the second procedure. During the first stage, the flap was only partially sutured to the recipient site to avoid
TABLE 1 Demographics and surgical details

\begin{tabular}{|c|c|}
\hline & Number \\
\hline \multicolumn{2}{|l|}{ Gender } \\
\hline Male & 42 \\
\hline Female & 11 \\
\hline Total & 53 \\
\hline \multirow[t]{2}{*}{ Age } & Mean: 65 years \\
\hline & Range: (45-72) \\
\hline \multirow[t]{2}{*}{ Follow-up } & Mean: 14 months \\
\hline & Range: (8-21) \\
\hline Diagnosis & Oral cancer (all patients) \\
\hline \multicolumn{2}{|l|}{ Predisposing factors } \\
\hline Tobacco & 49 \\
\hline Betel nut & 43 \\
\hline Alcohol & 37 \\
\hline \multicolumn{2}{|l|}{ Risk factors } \\
\hline Previous procedures & 41 \\
\hline Prior radiotherapy & 35 \\
\hline Recurrent cancer & 31 \\
\hline \multicolumn{2}{|l|}{ Location } \\
\hline Cheek & 49 \\
\hline Oral mucosa & 53 \\
\hline Tongue & 13 \\
\hline Lip & 5 \\
\hline Neck & 2 \\
\hline \multicolumn{2}{|l|}{ Defect size } \\
\hline & Mean: $96 \mathrm{~cm}^{2}$ \\
\hline & Range: $56-120 \mathrm{~cm}^{2}$ \\
\hline \multicolumn{2}{|l|}{ Flaps } \\
\hline $\mathrm{ALT}$ & 40 \\
\hline$A L T+V L$ & 13 \\
\hline \multicolumn{2}{|l|}{ Recipient artery } \\
\hline Superior thyroid & 30 \\
\hline Transverse cervical & 19 \\
\hline Thoracoacromial & 4 \\
\hline \multicolumn{2}{|l|}{ Recipient vein } \\
\hline External jugular & 42 \\
\hline Internal jugular and branches & 11 \\
\hline
\end{tabular}

any tension, torsion, or compression. Vital structures such as bone and vessels were covered in the first operation. If necessary, a temporary orocutaneous fistula was left in the first operation. The flap was allowed to stabilize over a period of 3-4 weeks. Flap stabilization was achieved through neovascularization from the surrounding tissue via the designed "anchoring points" that remained intact in the second procedure (Figure 1). Once swelling had subsided, the flap was partially elevated and divided into separate flaps. Then each part of the flap was rearranged on its corresponding pedicle based on the reconstructive needs while connections to the surrounding tissue remained intact via the anchoring points. Finally, the parts of the flap were inset completely to achieve the desired functional and aesthetic results. 


\section{Flap Harvest}
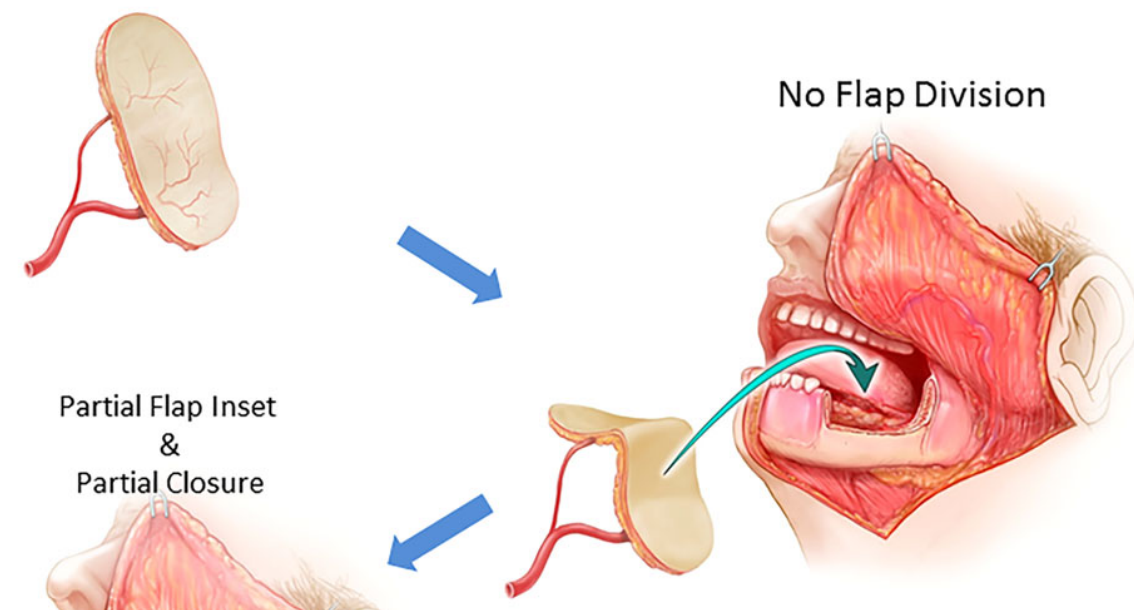

Partial Flap Inset

\&
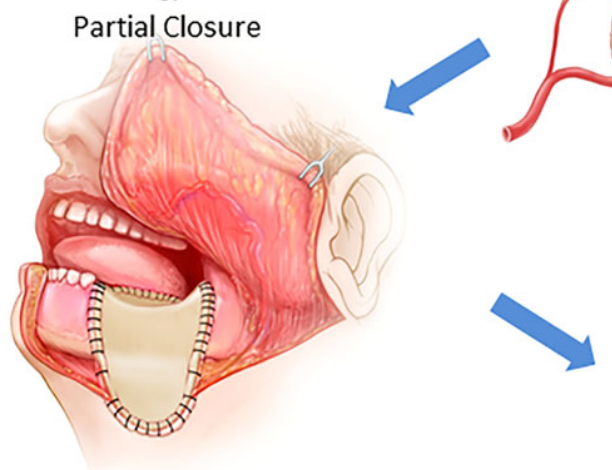

Final Closure
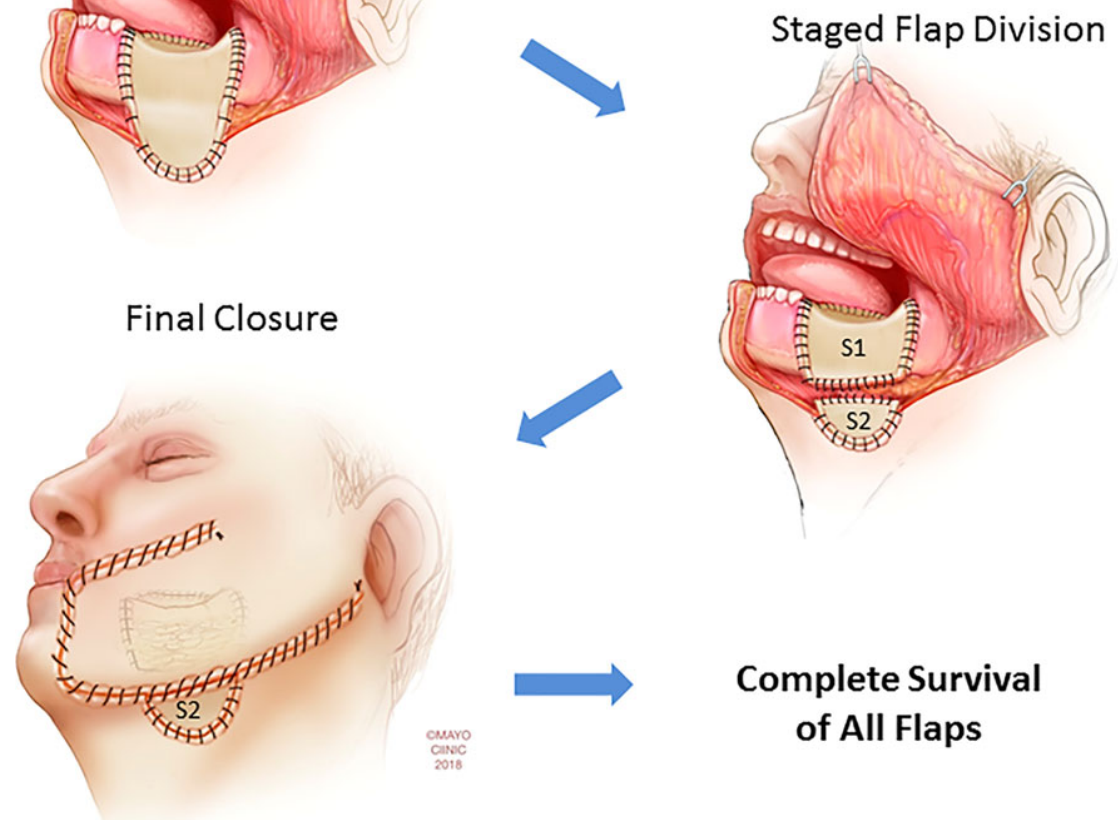

\section{Complete Survival of All Flaps}

FIGURE 1 The staged inset principle. Instead of primary division and complete inset, the flap received a partial inset initially with a partial closure of recipient site to avoid tension. The flap was allowed to stabilize over a period of time (3-4 weeks) before it was divided and partially mobilized for complete inset

\section{3 | RESULTS}

Out of the 53 cases that underwent staged inset, 47 had uneventful recovery with complete survival of the flaps (Table 2). Six patients required immediate re-exploration in the post-operative period due to signs of vascular compromise (changes in flap color and turgor, delayed capillary refill, decreased temperature). Of these, in 4 cases, the circulatory disturbance was secondary to swelling and was improved by releasing sutures and reinsetting the flap with no tension. To the contrary, despite revision of the vascular anastomosis, the remaining 2 flaps were unsalvageable. Most likely, this occured due to small caliber of the perforators. The flap survival rate was $96.2 \%$ (51 of 53 flaps). No flap component was lost after the second stage of inset. With the exception of 3 cases of wound infection that were managed successfully with excision and debulking in the second stage, final inset was successful in all cases (Table 2). The average time between the 2 surgical stages was 24 days (range, 21-28), mean hospital stay was 34 days (range, 29-36). Mean follow-up was 14 months, with a range of 8-21 months (Table 1). At the time of their last follow-ups, one patient had trismus but was unwilling to undergo further surgeries due to old age (72 years old). Another patient developed an orocutaneous fistula that lead to salivary leakage. It was treated with closure of the fistula. No patient had oral incontinence, and the remaining 49 patients had no associated complications and reported adequate oral function.

\section{1 | Case report}

A 61-year-old male presented with a painful ulcer on the right buccal mucosa for more than 4 months. Upon examination, the patient had 
TABLE 2 Postoperative details and outcomes

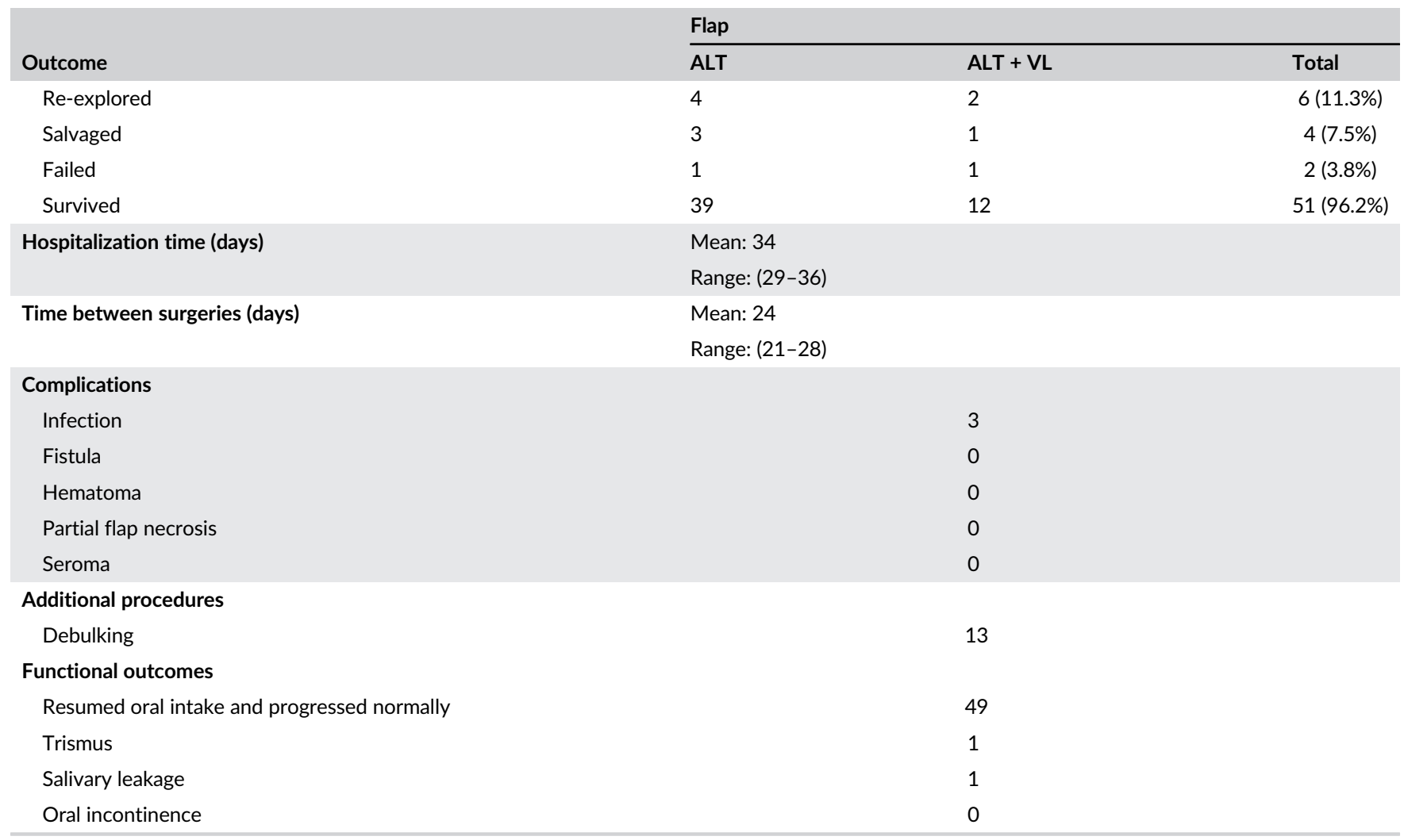

trismus with a maximum interincisal opening (MIO) of $18 \mathrm{~mm}$ due to panoral submucosal fibrosis. An ulcerative $4 \times 3 \mathrm{~cm}$ lesion was noted at the right posterior buccal mucosa. Biopsy of the lesion confirmed squamous cell carcinoma (SCC). Panorex imaging showed a generalized alveolar bone resorption. Subsequently, a composite resection of the tumor with marginal mandibulectomy and radical neck dissection was performed, creating a through-and-through cheek defect (Figure 2A,B). To reconstruct the oral and check defects, a flap measuring $10 \times 25 \mathrm{~cm}$ was planned on the left ALT. Two perforators from the lateral femoral circumflex artery (LFCA) were identified with handheld Doppler. However, the perforators were found to be very small and therefore a tensor fascia lata (TFL) flap was included in the flap. The recipient vessels were the right superior thyroid artery and the external jugular vein. Initially, the facial skin on the right side was elevated (Figure $2 \mathrm{~B}$ ) to allow free flap inset. The proximal part of the flap received a complete inset with the skin paddle covering the mucosal side. The flap was folded as an upside down "U" with the distal skin paddle covering the extraoral facial defect and exposed mandible. Then, the elevated facial skin was allowed to drape over the free flap. The distal part of the flap received partial inset with sutures only around half of the extraoral defect and to the lower face to secure the flap in place (Figure 2C,D). The lower face was partially closed with an opening left to avoid tension on the flap. Three weeks later (Figure 2E,F), the wound was revised though the previous midline incision on the chin. The flap was debulked by removing a portion of the middle (bridging) part of the skin flap, thus partially dividing the flap into 2. The distal part of the flap was further trimmed and deepithelialized to allow a complete closure of the wound (Figure 2E,F).
At 1-year follow-up, complete survival of the flap was noted with no contractures at the recipient site. The patient reported no salivary leakage or trismus of the mouth (Figure $2 \mathrm{G}, \mathrm{H}$ ).

\section{4 | DISCUSSION}

Herein, we present a safe strategy to approach complex head and neck reconstruction with free tissue transfer. The principle of staged inset should be considered early when flap or recipient site related risks are suspected (Figure 3). If the perforators are considerably small in caliber, the vascularity of the flap may be tenuous. The problem is even more pronounced in the presence of peripheral arterial occlusive disease (PAOD) with impaired blood flow in the distal arterial tree. In combination with tissue ischemia, the result may be a chronic nonhealing wound (Eweida, Lang, Schmitz, \& Horch, 2013) This concern is even more pertinent when a larger flap is required in order to meet the soft tissue requirements of an extensive defect. By maintaining a reliable pedicle and avoiding any compression, staged inset of flaps may allow optimal blood supply until the flap develops neovascularization from the surrounding tissues. This is particularly true in the case of a poor/sub-optimal recipient site such as irradiated tissue that, due to its impaired vascularity, may increase the risk of flap failure and wound infection (Halle et al., 2009) The staged inset is especially useful in reconstruction of complex, composite tissue defects. Torsion or tension on the flap components may result from folding and twisting during flap inset. Perforator kinking or stretching may also cause impaired vascularity (Chang, 2013; Gong et al., 2015; Kuo et al., 2010). With the proposed method, the flap can be inset at a 

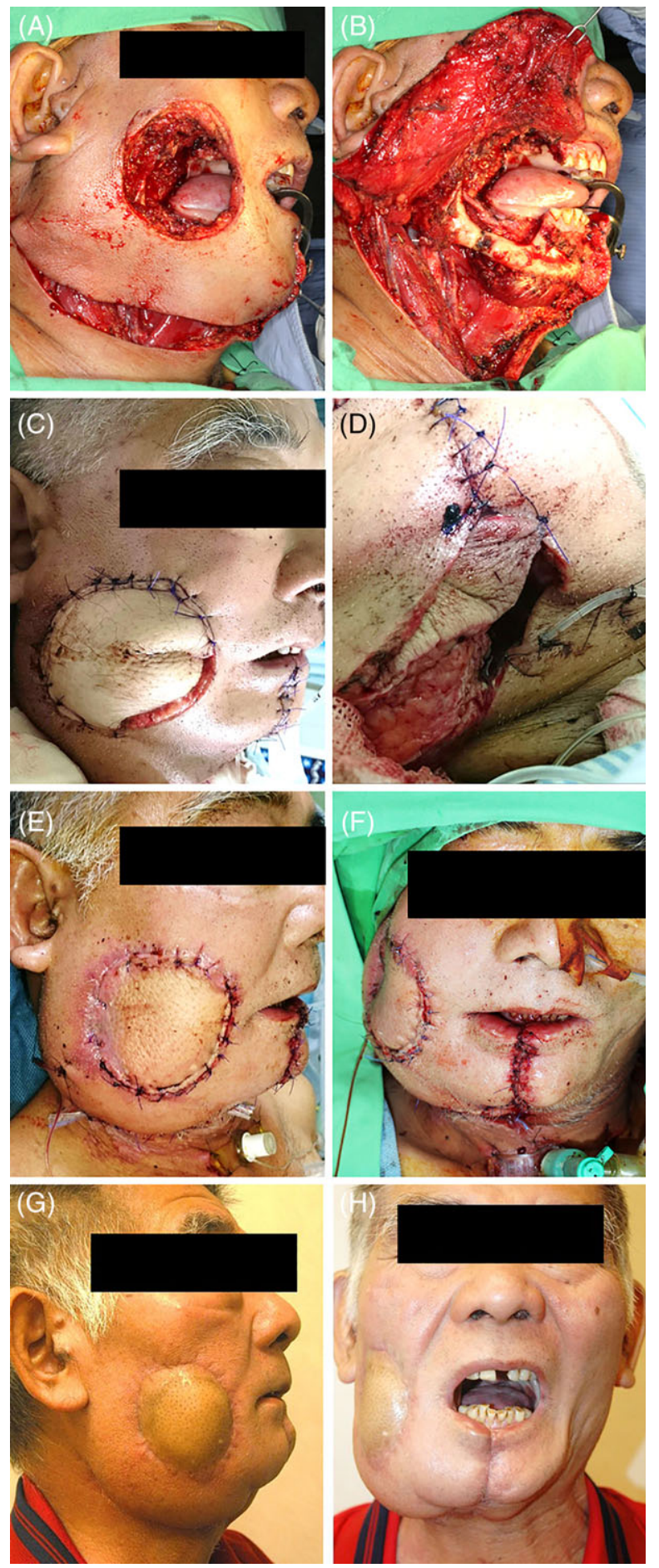

FIGURE 2 Case report. A, A through-and-through right cheek defect was present after composite resection and radical neck dissection for an oral tumor. B, The extent of resection and dissection could be seen. C, Immediately after the initial reconstruction, the flap was folded into an upside-down " $U$ ". The distal portion of the flap that covered the cheek received a partial inset (only half of the through-and-through defect was sutured) to secure the flap. The proximal and intraoral part of the flap was inset completely to avoid saliva leakage. D, The neck wound was partially closed with an area that was left open to avoid too much compression on the folded flap. E, F, 3 weeks later, during the second stage of the reconstruction, the flap was partially divided and deepithelialized to allow complete inset and closure of the wound. G, 1year postop. A lateral view of the patient that showed complete survival of the extraoral part of the flap and overall acceptable aesthetic appearance. $\mathrm{H}$, The patient did not have trismus postoperatively temporary relaxed position while neovascularization is being established. When a flap is fitted into a limited space such as the oral cavity, vascular supply to the flap and circulation within the flap itself may be compromised as a result of compression to the flap and/or its perforators. Consequently, partial or complete flap loss may occur due to the initial venous congestion followed by arterial insufficiency. To increase the chance of total flap survival, the cavity or wound can be first closed partially (Figure 2 ) and then completely covered at a second stage.

Although our success rate for this series was $96.2 \%$, it is important to keep in mind that this was a special group of patients with an increased risk of vascular compromise from over a 6-year period. Most patients had previous tumor resections and reconstructions often with adjuvant radiotherapy prior to referral to our center. Due to tumor recurrence, patients required further resections of parts of the cheek, oral lining, lips, and tongue (Table 1). All patients had SCC of the oral cavity mainly from long-term additive effects of betel quid, alcohol, and cigarette smoking. Their combined usage results in larger areas of SCC in the entire oral cavity attributed to the effect of field cancerization (Liao et al., 2014; Van Oijen \& Slootweg, 2000) which describes a process in which a broad area of tissues undergoes carcinogenic transformation. In addition, betel quid, alcohol, and cigarette smoking have been linked to not only a high incidence but also recurrence of oral cancer (Adel, Liao, \& Lee, 2016; Guo et al., 2013).

The strategy of staged flap inset has several advantages over the conventional method where more than one flaps are harvested in one operation. First of all, the surgical time is greatly reduced and only one set of recipient vessel is required. Although a second surgery is required, it is much shorter and simpler. The flap can be partially elevated, divided, and fitted into the target site during this stage. On the other hand, primary flap division and inset is the preferred method in a typical noncomplicated case, when both the donor and recipient sites are in optimal condition with decent perforator size and minimal risk of infection.

Additional factors to be considered when deciding on a staged inset in the first operation include bulky flaps harvested from obese patients and reconstructions involving the oral cavity which has a limited space. Complete inset can be attempted first and the flap assessed prior to final closure. If there is any sign of vascular compromise (impaired arterial inflow or venous congestion), one should perform a partial inset and plan staged inset to avoid tension.

In contrast to a regular revision, a staged flap inset is different as the time between the first and second operation is much shorter-3-4 weeks versus 2-3 months in a regular revision. In regular revision, the flap's vascular supply is no longer reliant on the original pedicle. On the other hand, in staged flap inset, the time window of 3-4 weeks allows the flap components to establish some neovascularization from the surrounding tissues, but the original pedicle is still an important source of vascular supply to the flap components. It is this combination of these vascular supplies that greatly increases the chances of total survival of the flap components. A study in a swine model has shown that neovascularization between the flap and the surrounding tissue becomes well established by the 8th day after free tissue transfer (Black, Chait, O'Brien, 


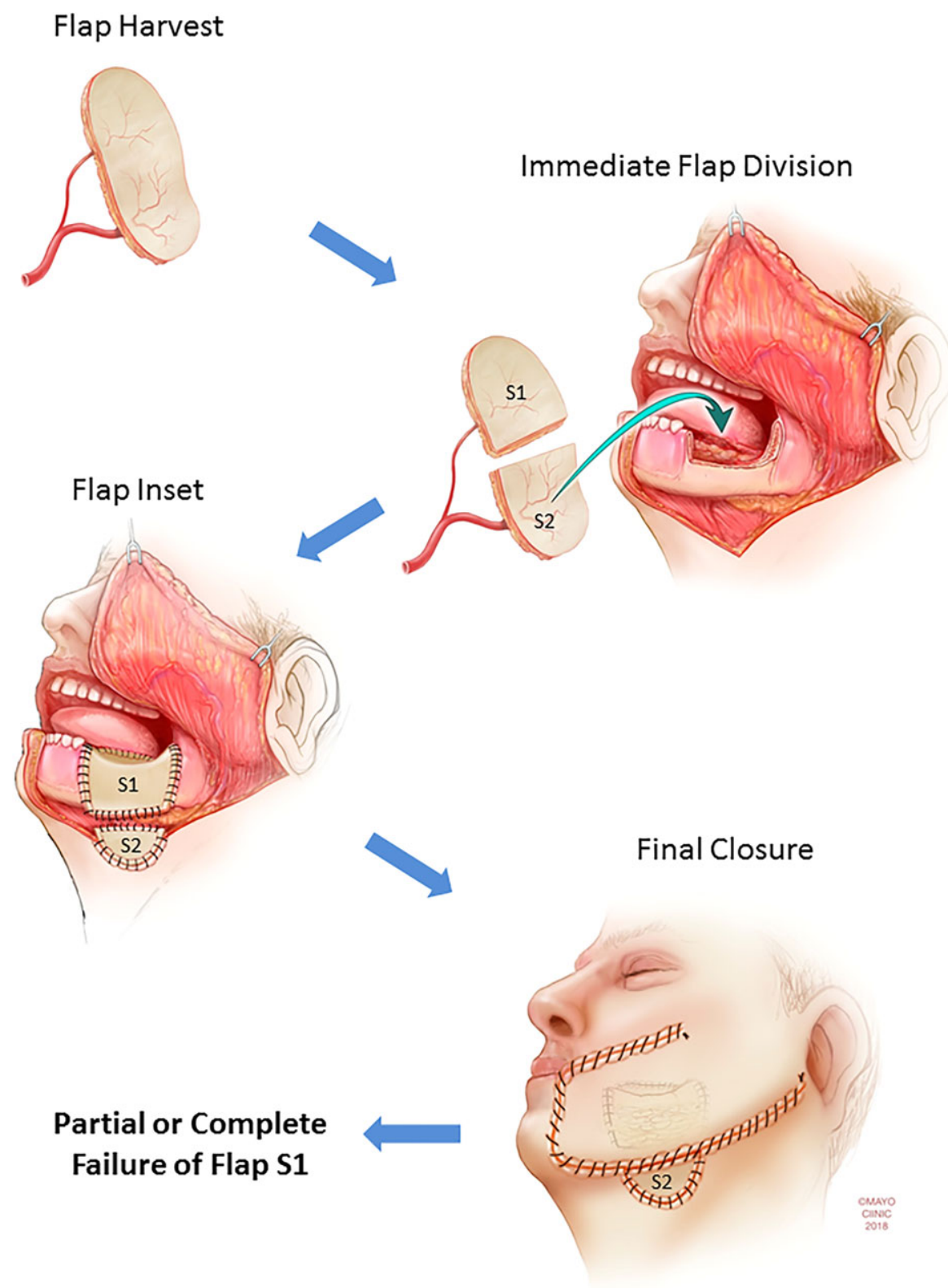

FIGURE 3 An illustration showing the conventional single stage flap inset. After harvest, a flap could be completely divided to meet the reconstructive demand. However, in case of unfavorable conditions such as small caliber perforators, postradiotherapy, and high risk of infection at the recipient site, primary division and complete inset might result in complete or partial flap loss

Sykes, \& Sharzer, 1978) and serves as the basis for the staged inset principle. A clinical study determined that free flaps are able to survive a pedicle occlusion only after postoperative day 10 (Granzow, Li, Caton, \& Boyd, 2015; Yoon \& Jones, 2016) Another case series that investigated the effect of early pedicle loss documented that $50 \%$ of their flaps were able to survive completely after the loss of arterial inflow on the postoperative day 7 (Salgado et al., 2002) However, considering the complexity of head and neck procedures and confounding risk factors such as preoperative radiotherapy and scarring of the recipient site tissue bed, a period of 3-4 weeks is preferred. An advantage of the staged flap inset is the much shorter and simpler operation of the second procedure. A disadvantage would be that if a major rearrangement is required (i.e. out of reach of the original pedicle), a regular revision is more suitable, which is typically done at a much later time.
When staged flap inset is used for through-and-through cheek defects, the intraoral side should be given priority for complete flap inset during the first operation to cover any exposed bone, hardware, vessels, or nerves, whereas the extraoral side can be partially inset to avoid vascular compromise. The goal is to reduce the chance of infection and saliva leakage (Liu, Yang, \& Ou, 2011) in addition to minimizing the risk of partial flap loss.

The staged inset protocol is longer than most conventional protocols and may lead to a delay in patient management. However, it is necessary to emphasize that this procedure is uncommon even at our unit (53 cases over a 6-year period), as we always strive to accomplish inset in a single if it is possible and safe. This procedure is reserved for select patients with high risk of flap failure (thick flap, unfavorable perforator characteristics, and tight-fitting recipient site) who nevertheless require reconstruction. Many of the 
selected patients had previous failed reconstruction attempts and were referred from other centers. In some cases, the 2-stage flap inset was chosen after an attempt at one-stage was noted to be failing intraoperatively. Staged flap inset is safer and it can maximize the chance of total flap survival, which is critical, as many of these patients may require adjuvant chemoradiotherapy following the reconstruction. Moreover, the aforementioned cancer treatments may result in dramatic changes in the flap, making the survival of every part of the flap even more necessary. Additionally, in our patient cohort, the decision to keep a patient hospitalized was also based on thorough evaluation of the patient's social and living arrangement in order to determine if the patient can be properly taken care of once discharged. Due to safety concerns, only simple division of the flap was done for the frail elderly oncological patients to obtain an acceptable appearance without further remodeling.

Often, it is difficult to make the decision to perform a staged flap inset instead of a typical primary inset as it is dependent on the surgeon's experience in predicting complications. Switching to a staged inset is recommended upon suspecting any chance of partial or complete flap failure. Complete survival of all flap components is the basis for successful complex free flap reconstruction. Over time, partial flap failure or tenuous vascular supply may cause atrophy of the transferred tissue, resulting in contracture or trismus. The novelty of this method is that it offers a safer approach to a free flap head and neck reconstruction that is otherwise associated with a high chance of failure.

\section{5 | CONCLUSION}

Although staged flap inset requires a 2-step surgical approach, this experience shows that it is a safe technique that can improve the chance of complete flap survival in a cohort of patients with an otherwise high risk of flap failure. Selective application of this technique is fundamental for optimum outcomes.

\section{ACKNOWLEDGMENTS}

The authors would like to express their gratitude to Dr. Hung-Yang Lin for providing excellent photographs to be used in this manuscript and Michael King for the beautiful illustrations. All authors contributed equally to the work of this article.

\section{ORCID}

Tony C.T. Huang (D) https://orcid.org/0000-0003-0462-7786

Pedro Ciudad (D) https://orcid.org/0000-0003-4646-2676

Oscar J. Manrique (D) https://orcid.org/0000-0001-9688-3079

Mouchammed Agko (iD https://orcid.org/0000-0003-0242-0941

M. Diya Sabbagh (D) https://orcid.org/0000-0002-1900-7574

\section{REFERENCES}

Adel, M., Liao, C.-T., Lee, L.-Y., Hsueh, C., Lin, C. Y., Fan, K. H., et al. (2016). Incidence and outcomes of patients with oral cavity squamous cell carcinoma and fourth primary tumors: A long-term follow-up study in a betel quid chewing endemic area. Medicine (Baltimore), 95(12), e2950.

Black, M. J., Chait, L., O'Brien, B. M., Sykes, P. J. J., \& Sharzer, L. A. A. (1978). How soon may the axial vessels of a surviving free flap be safely ligated: A study in pigs. British Journal of Plast Surgery, 31(4), 295-299.

Chana, J. S., \& Wei, F.-C. (2004). A review of the advantages of the anterolateral thigh flap in head and neck reconstruction. British Journal of Plastic Surgery, 57(7), 603-609.

Chang, E. I. (2013). My first 100 consecutive microvascular free flaps. Plastic and Reconstructive Surgery, 1(4), e27.

Eweida, A. M., Lang, W., Schmitz, M., \& Horch, R. E. (2013). Salvage of a free radial forearm flap by creation of an arteriovenous fistula at the distal arterial pedicle. Microsurgery, 33(5), 391-395.

Gong, Z., Zhang, S., Wang, K., Tan, H. Y., Zhu, Z. F., Liu, J. B., ... Wu, H. J. (2015). Chimeric flaps pedicled with the lateral circumflex femoral artery for individualised reconstruction of through-and-through oral and maxillofacial defects. The British Journal of Oral \& Maxillofacial Surgery, 53(2), 148-152.

Granzow, J., Li, A. I., Caton, A., \& Boyd, J. B. (2015). Free flap survival following failure of the vascular pedicle. Annals of Plastic Surgery, 75(1), 44-48.

Guo, S., Huang, T., Huang, J., Lin, M., Hong, R., Chang, C., \& Chen, M. (2013). Alcohol, betel-nut and cigarette consumption are negatively associated with health promoting behaviors in Taiwan: A cross-sectional study. BMC Public Health, 13(1), 1.

Halle, M., Bodin, I., Tornvall, P., Wickman, M., Farnebo, F., \& Arnander, C. (2009). Timing of radiotherapy in head and neck free flap reconstruction-A study of postoperative complications. Journal of Plastic, Reconstructive \& Aesthetic Surgery, 62(7), 889-895.

Huang, W.-C., Chen, H.-C., Jain, V., Kilda, M., Lin, Y. D., Cheng, M. H., ... Wei, F. C. (2002). Reconstruction of through-and-through cheek defects involving the oral commissure, using chimeric flaps from the thigh lateral femoral circumflex system. Plastic and Reconstructive Surgery, 109(2), 433.

Huang W.-C., Chen, H.-C., Wei, F.-C., Cheng, M.-H., \& Schnur D. P. (2003). Chimeric flap in clinical use. Clinics in Plastic Surgery, 30(3), 457-467.

Jeng, S.-F., Kuo, Y.-R., Wei, F.-C., Su, C.-Y., \& Chien, C.-Y. (2005). Reconstruction of extensive composite mandibular defects with large lip involvement by using double free flaps and fascia lata grafts for oral sphincters. Plastic and Reconstructive Surgery, 115(7), 1830-1836.

Louise Kent, M. L., Brennan, M. T., Noll, J. L., Fox, P. C., Burri, S. H., Hunter, J. C., \& Lockhart, P. B. (2008). Radiation-induced trismus in head and neck cancer patients. Support Care Cancer, 102(3), 338.

Khouri, R. K., Cooley, B. C., Kunselman, A. R., Landis, J. R., Yeramian, P., Ingram, D., ... Wallemark, C. (1998). A prospective study of microvascular free-flap surgery and outcome. Plastic and Reconstructive Surgery, 102(3), 711-721.

Kuo, Y.-R., Shih, H.-S., Chen, C.-C., Boca, R., Hsu, Y. C., Su, C. Y., ... Wei, F. C. (2010). Free fibula osteocutaneous flap with soleus muscle as a chimeric flap for reconstructing mandibular segmental defect after oral cancer ablation. Annals of Plastic Surgery, 64(6), 738-742.

Kuzon, W. M., Jejurikar, S., Wilkins, E. G., \& Swartz, W. M. (1998). Double free-flap reconstruction of massive defects involving the lip, chin, and mandible. Microsurgery, 18(6), 372-378.

Liao, C. T., Wallace, C. G., Lee, L. Y., Hsueh, C., Lin, C. Y., ... Yen, T. C. (2014). Clinical evidence of field cancerization in patients with oral cavity cancer in a betel quid chewing area. Oral Oncology, 50(8), 721-731.

Liu, W. W., Yang, A. K., \& Ou, Y. D. (2011). The harvesting and insetting of a chimeric anterolateral thigh flap to reconstruct through and through cheek defects. International Journal of Oral and Maxillofacial Surgery, 40(12), 1421-1423.

Pohlenz, P., Blessmann, M., Heiland, M., Blake, F., Schmelzle, R., \& Li, L. (2007). Postoperative complications in 202 cases of microvascular head and neck reconstruction. Journal of Cranio-Maxillofacial Surgery, 35(6-7), 311-315. 
Salgado, C. J., Smith, A., Kim, S., Higgins, J., Behnam, A., Herrera, H. R., \& Serletti, J. M. (2002). Effects of late loss of arterial inflow on free flap survival. Journal of Reconstructive Microsurgery, 18(7), 579-583.

Van Oijen, M. G., \& Slootweg, P. J. (2000). Oral field cancerization: carcinogen-induced independent events or micrometastatic deposits? Cancer Epidemiology Biomarkers Prevention, 9, 249-256.

Wang, C. J., Huang, E. Y., Hsu, H. C., Chen, H. C., Fang, F. M., \& Hsiung, C. Y. (2005). The degree and time-course assessment of radiation-induced trismus occurring after radiotherapy for nasopharyngeal cancer. The Laryngoscope, 115, 1458-1460.

Yoon, A. P., \& Jones, N. F. (2016). Critical time for neovascularization/angiogenesis to allow free flap survival after delayed postoperative anastomotic compromise without surgical intervention: a review of the literature. Microsurgery, 36, 604-612.

How to cite this article: Huang TCT, Ciudad P, Manrique OJ, et al. Staged inset of free flaps for complex microsurgical head and neck reconstruction to ensure total flap survival. Microsurgery. 2018;38:844-851. https://doi.org/10.1002/micr.30370 